\title{
Germline mutation contribution to chromosomal instability
}

\author{
Sock Hoai Chan' and Joanne Ngeow ${ }^{1,2}$ \\ 'Division of Medical Oncology, Cancer Genetics Service, National Cancer Centre Singapore, Singapore \\ 2Oncology Academic Clinical Program, Duke-NUS Medical School Singapore, Singapore
}

Correspondence should be addressed to J Ngeow

Email

Joanne.Ngeow.Y.Y@ singhealth.com.sg

\begin{abstract}
Genomic instability is a feature of cancer that fuels oncogenesis through increased frequency of genetic disruption, leading to loss of genomic integrity and promoting clonal evolution as well as tumor transformation. A form of genomic instability prevalent across cancer types is chromosomal instability, which involves karyotypic changes including chromosome copy number alterations as well as gross structural abnormalities such as transversions and translocations. Defects in cellular mechanisms that are in place to govern fidelity of chromosomal segregation, DNA repair and ultimately genomic integrity are known to contribute to chromosomal instability. In this review, we discuss the association of germline mutations in these pathways with chromosomal instability in the background of related cancer predisposition syndromes. We will also reflect on the impact of genetic predisposition to clinical management of patients and how we can exploit this vulnerability to promote catastrophic genomic instability as a therapeutic strategy.
\end{abstract}

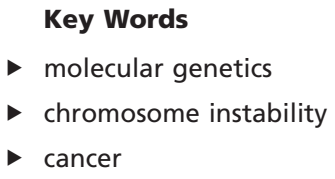

Endocrine-Related Cancer (2017) 24, T33-T46

\section{Introduction}

Genomic instability is a hallmark of human cancers (Negrini et al. 2010). It refers to the increased frequency of accruing genetic alterations, ranging from single nucleotide mutations to whole chromosome changes such as aneuploidy (Pikor et al. 2013, Giam \& Rancati 2015) and can be broadly viewed in three main groups. Nucleotide instability (NIN) is the increased frequency of base-pair mutations (Negrini et al. 2010, Pikor et al. 2013), whereas microsatellite instability (MIN) is characterized by the expansion and contraction of microsatellite sequences, usually as a consequence of defective cellular DNA mismatch repair (Boland \& Goel 2010, Negrini et al. 2010, Pikor et al. 2013). However, the most prominent form of genomic instability in human cancers is chromosomal instability (CIN), which is the elevated rate of gain or loss of segmental or whole chromosomes, typically resulting in karyotypic heterogeneity (Negrini et al. 2010, Printed in Great Britain
Pino \& Chung 2010, Bakhoum \& Compton 2012, Pikor et al. 2013). Most solid tumors display some form of aneuploidy, and it has been long implicated that CIN fuels cancer development and progression (Negrini et al. 2010, Bakhoum \& Compton 2012).

Hereditary cancers, or cancer-predisposing syndromes, are associated with inherited germline mutations that increase an individual's lifetime risk of cancer (Garber \& Offit 2005). One example is the hereditary non-polyposis colon cancer (HNPCC), also known as Lynch syndrome (OMIM 120435). Up to 70\% of HNPCC families have germline mutations in DNA mismatch repair genes MLH1, MSH2 or MSH6, leading to MIN (Lengauer et al. 1998, Garber \& Offit 2005, Boland \& Goel 2010). In the majority of families meeting criteria for Li-Fraumeni syndrome (OMIM 151623), germline mutations in TP53 are frequently observed (Garber \& Offit 2005). In another

This paper forms part of a special section on 50 Years of Tubulin. The Guest Editors for this section were Karen Crasta and Ritu Aneja. 
instance, as many as 50\% of hereditary breast and ovarian cancers (HBOC, OMIM 604370) are associated with mutations in BRCA genes, which are known to have important functions in DNA double-strand breaks (DSBs) repair (Venkitaraman 2004, Negrini et al. 2010). A unifying characteristic of these hereditary cancers is the early manifestation of malignancy, usually in childhood or early adulthood. In the framework of Knudson's twohit hypothesis, harboring a germline mutation in highly penetrant genes such as TP53 or BRCA1 increases the risk for earlier tumor development as less number of events are required for initiation of tumorigenesis (Knudson 1996, 2001) (Table 1). CIN is a feature in the tumors associated with these hereditary cancers (Negrini et al. 2010), and it is conceivable that germline mutations affecting the genes involved in maintaining genomic stability may predispose to higher likelihood of CIN, and consequently, an elevated chance of loss of heterozygosity ( $\mathrm{LOH})$ of critical tumor suppressors or amplifying oncogenes via chromosomal mis-segregation, and therefore, an overall increased risk for cancer (Kops et al. 2005). However, it still remains an on-going discussion whether germline mutations in any candidate gene actually predisposes to CIN. In this review, we discuss the emerging evidences for contribution of germline mutations to genomic instability, with a focus on CIN, and the association to cancer predisposition.

\section{Chromosomal instability (CIN)}

CIN is characterized by the increased frequency of chromosome mis-segregation. CIN tumors commonly feature wide-spread changes in chromosome number also known as aneuploidy, translocations, inversions, amplifications and deletions, often involving the loss of heterozygosity (LOH) (Pino \& Chung 2010, Schvartzman et al. 2010, Pikor et al. 2013). Populations of CIN cells with such karyotypically variable genome are capable of selective evolution and under the right conditions, can subsequently undergo clonal expansion thus driving tumor development, progression as well as acquisition of resistance to therapy (Lengauer et al. 1998, Bakhoum \& Compton 2012, Pikor et al. 2013).

The molecular mechanisms underlying CIN has been actively investigated for years and we now know these include defects in mitotic spindle assembly checkpoint (SAC), sister chromatid cohesion, kinetochoremicrotubule attachment dynamics, cell cycle regulation, multipolar spindles as well as supernumerary centrosomes
(Thompson et al. 2010, Bakhoum \& Compton 2012, Giam \& Rancati 2015). Under normal circumstances, proper functioning of these mechanisms govern the fidelity of chromosome segregation by ensuring correct orientation of all chromosomes on the microtubule spindle for cell division and deferring progression into the anaphase stage of mitosis until the chromosomes are ready for division (Holland \& Cleveland 2009, Thompson et al. 2010, Bakhoum \& Compton 2012). Defects in these mechanisms lead to chromosome lagging as well as premature initiation of anaphase before all the chromosomes are aligned to the spindles, leading to an increased risk of chromosome mis-segregation (Fig. 1).

\section{Germline mutations affecting SAC}

One of the thoroughly investigated mechanisms contributing to CIN is the mitotic SAC. The intricacies of SAC are reviewed in detail elsewhere (Lara-Gonzalez et al. 2012, Foley \& Kapoor 2013, Musacchio 2015). Essentially, the SAC monitors and ensures that all chromosomes are properly attached by their kinetochores to spindle microtubules so that chromosome segregation can proceed accurately in order to maintain integrity and stability of the cellular genome. Unattached or incorrectly attached chromosomes lead to the assembly of the mitotic checkpoint complex (MCC), triggering the SAC signaling, which inhibits activity of the E3 ubiquitin ligase anaphase-promoting complex/cyclosome (APC/C) and ultimately preventing entry into anaphase. MCC, the effector of SAC, is a complex of proteins including MAD1, MAD2, MPS1, BUB1, BUB3, BUBR1 and CDC20. Mutations in the genes encoding components of MCC lead to impairment of SAC signaling, thereby increasing the likelihood of chromosome mis-segregation and CIN (Thompson et al. 2010). This is demonstrated by mosaic variegated aneuploidy syndrome, a rare disorder associated with germline mutations in $B U B 1 B$, an SAC gene encoding BUBR1 of the MCC.

Mosaic variegated aneuploidy syndrome (MVA, OMIM 257300) is an autosomal recessive disorder marked by constitutional CIN in the form of mosaic aneuploidies, mostly trisomies and monosomies. Clinically, MVA patients typically present with growth retardation, microcephaly, variable developmental delay, a broad spectrum of congenital abnormalities and a higher risk of cancer, particularly childhood malignancies such as rhabdomyosarcoma, Wilms' tumor and leukemia (Hanks et al. 2004, Schvartzman et al. 2010).
C 2017 Society for Endocrinology Printed in Great Britain
Published by Bioscientifica Ltd 
Table 1 Cancer predisposition syndromes commonly associated with genomic instability.

\section{Cancer predisposition syndrome}

Mosaic variegated aneuploidy syndrome

Premature chromatid separation syndrome

Familial adenomatous polyposis

Hereditary nonpolyposis colorectal cancer/Lynch syndrome

Hereditary breast and ovarian cancer
227650

Fanconi anemia
OMIM no.

Ataxia-telangiectasia

Nijmegen breakage syndrome

Bloom syndrome

Werner syndrome
604370

612555

257300

176430

175100

CIN

120435

MIN

CIN

CIN

Associated type of

genomic instability

CIN

CIN

CIN

251260

CIN

210900

CIN

277700
CIN
Reported tumor risk

Rhabdomyosarcoma

Wilms tumor

Leukemia

Colon cancer

Stomach cancer

Seminoma

Keratoacanthoma

Carcinoma in situ of uterine

cervix

Desmoid tumors

Fibrosarcoma

Colorectal cancer

Endometrial cancer

Ovarian cancer

Gastric cancer

Hepatobiliary cancers

Small bowel cancers

Urothelial cancers

Prostate cancer

Pancreatic cancer

Breast cancer

Ovarian cancer

Prostate cancer

Pancreatic cancer

Melanoma

Leukemia

Head and neck cancer

Brain tumors

Wilms tumor

Breast cancer

Pancreatic cancer
Colorectal cancer

Known susceptibility

genes

BUB1B

BUB1B

$A P C$

MSH2

$M L H 1$

MSH6

PMS

RCA1

$B R C A 2$

FANCA

FANCB

FANCC

FANCD1 (BRCA2)

FANCD2

FANCE

FANCF

FANCG

FANCI

FANCJ (BRIP1)

FANCL

FANCM

FANCN (PALB2)

FANCO (RAD51C)

FANCP (SLX4)

FANCQ (ERCC4)

FANCS (BRCA1)

FANCR (RAD51)

FANCT (UBE2T)

ATM

Lymphoma

Sarcomas

Ovarian cancer

Breast cancer

Gastric cancer

Brain tumors

Lymphoma

Brain tumors

$N B N$

Rhabdomyosarcoma

Leukemia

Lymphoma

Wilms tumor

Colon cancer

Sarcomas

Thyroid cancer

Melanoma

Meningioma 
Germline mutations of $B U B 1 B$, which encodes BUBR1, were first reported in MVA families (Hanks et al. 2004). A combination of missense and truncating biallelic $B U B 1 B$ germline mutations were observed in five unrelated individuals with two presenting embryonal rhabdomyosarcoma at age 7 years and 5 months, respectively (Hanks et al. 2004), suggesting the compromised expression of BUBR1 may predispose to constitutional CIN, and therefore, increased risk of cancer. Monoallelic BUB1B germline mutations were initially reported in seven Japanese families with premature chromatid separation syndrome (PCS, OMIM 176430), a disorder phenotypically associated with MVA syndrome (Matsuura et al. 2006). Subsequent follow-up investigations showed that these families carried another germline intergenic missense mutation in $B U B 1 B$ that segregated with the disorder (Ochiai et al. 2014). Geneediting mediated functional analysis of the $B U B 1 B$ intergenic missense mutation demonstrated causality of this genetic change to PCS (MVA) syndrome (Ochiai et al. 2014). In addition to association with MVA, germline mutations in $B U B 1 B$ are also implicated in elevated risk for adult cancers. A germline homozygous intronic mutation (c. 2386-11A $\rightarrow \mathrm{G}$ ) in $B U B 1 B$ was identified in a patient with a history of multiple primary gastrointestinal cancers without any mutations detected in the known associated cancer predisposition genes MLH1, MSH2, MSH6 and PMS2, but whose cells demonstrated PCS and MVA (Rio Frio et al. 2010). More recently, whole-genome sequencing of 638 patients with familial pancreatic cancer (FPC) found a higher frequency of deleterious germline $B U B 1 B$ variants among FPC kindreds compared to control populations, providing support for $B U B 1 B$ as a candidate pancreatic cancer susceptibility gene (Roberts et al. 2016).

Collectively, most of these studies presented evidences that biallelic germline mutations of $B U B 1 B$ resulting in the reduced expression of BUBR1 are associated with constitutional CIN and that the loss of a fully functioning BUBR1 potentially predispose to a higher risk for cancer. Interestingly, a germline monoallelic deletion of approximately $1.75 \mathrm{Mb}$ affecting BUB1, another important component of MCC, was reported in a young microsatellite-stable colon cancer patient presenting increased constitutive aneuploidy, implying that haploinsufficiency of BUB1 may also contribute to early-onset gastrointestinal cancers (de Voer et al. 2011). The authors followed up by screening a larger population of 208 young (aged $<40$ years) patients with familial or early-onset colorectal cancer and found that germline mutations in BUB1 and BUB3 were associated with $2.9 \%$ of these patients, some of them who demonstrated variegated aneuploidies (de Voer et al. 2013). However, this was contradicted by findings in a study screening 192 patients with early-onset ( $<50$ years) colorectal cancer, which showed that $B U B 1 B$ germline mutation is not enriched in these patients thus is unlikely to have a major role in predisposition to colorectal cancer, although it was acknowledged that the sample size was too small to conclusively determine the role of $B U B 1 B$ in colorectal risk and that germline mutations in $B U B 1 B$ are associated with a variable phenotype (GarcíaCastillo et al. 2008) made complex by other risk factors such as environmental influence as well as other genetic modifiers (Hahn et al. 2016).

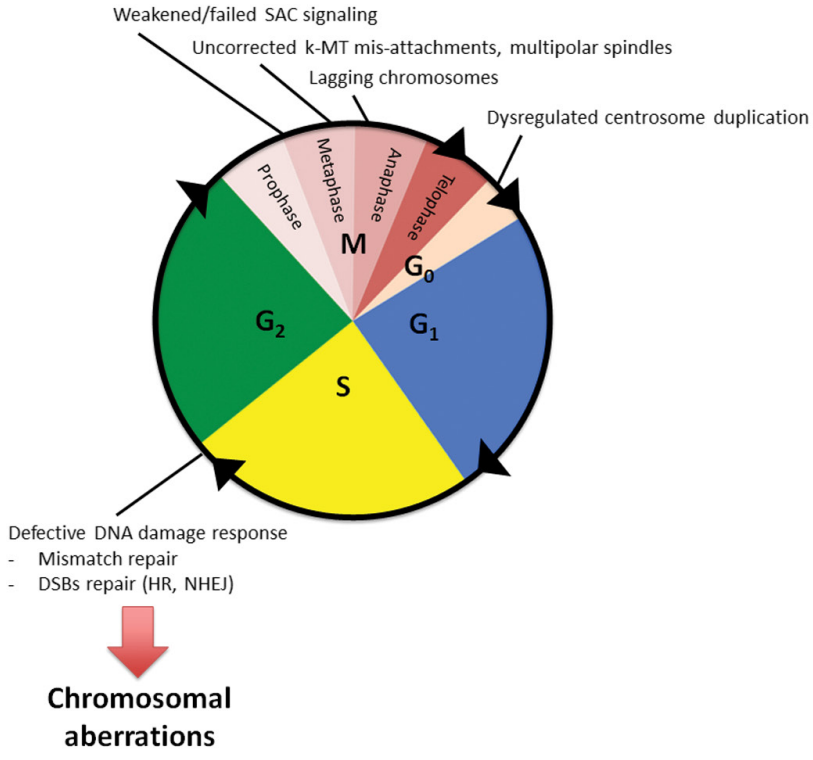

http://erc.endocrinology-journals.org DOI: 10.1530/ERC-17-0062
C) 2017 Society for Endocrinology Printed in Great Britain

\section{Chromosome} mis-segregation

Published by Bioscientifica Ltd.
Figure 1

Defects at cell cycle checkpoints in which germline mutations have been found to be associated with chromosomal instability. Defective DNA damage response and repair, particularly in the mismatch and DNA doublestrand breaks (DSBs) repair results in chromosomal aberrations such as deletions, amplifications, inversions and translocations. Impaired spindle assembly checkpoint (SAC) signaling, uncorrected kinetochore-microtubule (k-MT) mis-attachments, multipolar spindles due to supernumerary centrosomes resulting from dysregulated centrosome duplication contribute to lagging chromosomes in anaphase, resulting in chromosomal mis-segregation. These abnormalities combine to predispose the cell to chromosomal instability. HR, homologous recombination; NHEJ, non-homologous end joining. 
Apart from defective MCC, germline mutations in the genes that modulate recruitment of MCC components can also impair SAC signaling and may contribute predisposition to CIN. One such modulator is CENP-E, a kinetochore-associated kinesin-like protein that recruits BUBR1 to kinetochores as well as facilitating kinetochoremicrotubule interactions (Pino \& Chung 2010). Cells derived from $\mathrm{CENP}^{-\mathrm{E}^{+/}}$mice exhibited misaligned chromosomes with concomitant reduction in CENP-E expression and were found to develop significantly more chromosomally unstable cells in vitro over time (Weaver et al. 2007). The aneuploid CENP-E ${ }^{+/-}$cells demonstrated transformative properties and CENP-E $\mathrm{E}^{+/}$ mice were found to have a higher rate of spontaneous tumor development, specifically splenic lymphomas and lung adenomas, compared to wild-type littermates (Weaver et al. 2007).

\section{Germline mutations affecting kinetochore-microtubule dynamics}

The faithful segregation of chromosome during anaphase is dependent on the proper attachment of chromosome kinetochores to microtubule spindles. Normally, the kinetochores of sister chromatids of each chromosome engage in amphitelic attachment at metaphase, in which sister kinetochores attach to microtubules emanating from opposite spindles poles so that sister chromatids are pulled to opposing spindle poles during anaphase (Gregan et al. 2011). Merotelic attachment occurs when a single kinetochore is attached to microtubules from both spindle poles. This erroneous orientation is usually corrected prior entry into anaphase, failing which is known to result in lagging chromatids and chromosome mis-segregation (Thompson et al. 2010, Gregan et al. 2011, Santaguida \& Amon 2015).

Correction of the merotelic attachment is mediated by protein kinases, e.g. Aurora B and MPS1, via modification of the kinetochore-microtubule interaction dynamics (Musacchio 2015, Santaguida \& Amon 2015). As resolution of merotelic attachment into the proper amphitelic kinetochore orientation requires the release of improperly attached microtubules, hyperstable kinetochore-microtubule interactions can contribute to inefficient correction leading to a higher chance of incorrectly attached kinetochores entering anaphase and a predisposition to CIN (Thompson et al. 2010, Holland \& Cleveland 2012). It has been demonstrated that kinetochore-microtubules interactions of cancer cell lines with CIN have a significantly longer half-life and slower turnover rates (Bakhoum et al. 2009). This increased stability of kinetochore-microtubule interaction likely contributes to the decreased efficiency of correcting merotelic attachments and therefore a higher risk of CIN (Bakhoum et al. 2009, Thompson et al. 2010). For instance, partial inhibition of Aurora B kinase activity has been shown to suppress kinetochore-microtubule turnover during prometaphase, leading to increased frequency of merotelic attachments and chromosome lagging during anaphase (Cimini et al. 2006). A member of the Aurora family of Ser/Thr kinases, Aurora B, has a role in regulation of chromosome-microtubule interactions, chromatid cohesion as well as spindle stability (Carmena et al. 2009). Through its phosphorylation activities, Aurora B continuously destabilizes kinetochore-microtubule attachments until all kinetochores are properly bi-oriented (Carmena et al. 2009, Chan et al. 2012). Therefore, it is conceivable that constitutional depletion of Aurora B would affect kinetochore-microtubule dynamics resulting in impaired correction of merotelic attachments. Indeed, misaligned chromosomes as well as abnormal and multipolar spindles suggestive of defective bipolar spindle organization have been observed in cells derived from $\mathrm{Aurkb}^{+/-}$heterozygous mutant mice, with many of these mutants displaying a higher incidence of tumor formation, including pituitary and liver adenocarcinomas as well as skin papillomas (Fernández-Miranda et al. 2011). Although germline mutations in Aurora $B$ have not been reported in association with human cancer syndromes, it is not improbable that germline mutations affecting genes that regulate Aurora B activity, such as MAD2, would have the same effect of inhibiting the proper functioning of Aurora B in ensuring accurate chromosome segregation, thus predisposing to CIN and a higher risk of cancer (Cimini et al. 2006, Thompson et al. 2010, Schuyler et al. 2012, Shandilya et al. 2016).

The tumor suppressor protein APC, a known cancer predisposition of familial adenomatous polyposis (FAP, OMIM 175100), has also been implicated a role in chromosome segregation by affecting kinetochoremicrotubule interactions (Fodde et al. 2001, Kaplan et al. 2001, Aoki \& Taketo 2007, Rusan \& Peifer 2008, Bahmanyar et al. 2009, Bakhoum et al. 2009). Depletion of the APC in diploid cells has been shown to result in hyperstable kinetochore-microtubule attachments, accompanied by a significantly higher rate of lagging chromosomes at anaphase (Bakhoum et al. 2009). It has also been proposed that APC-depleted cells experience disruption of kinetochore-microtubule dynamics in the form of frequent pausing at the microtubule +end, which

Published by Bioscientifica Ltd. 
affects kinetochore oscillations, thus contributing to misorientation of the kinetochores, and consequently, predisposition to chromosomal mis-segregation (Draviam et al. 2006, Bahmanyar et al. 2009). In support of the contribution of APC to microtubule stability, APCdepleted cells were found to be more sensitive than wildtype cells to treatment of the microtubule-depolymerizing drug vinorelbine (Klotz et al. 2012), suggesting that disrupted microtubule dynamics is a feature of cells with impaired APC function and may be a potential therapeutic target for APC-deficient cancers. Apart from affecting kinetochore-microtubule interactions, loss of functional APC may also compromise the SAC through inefficient localization of BUB1 and BUBR1 to kinetochores as well as inappropriately activating the Wnt signaling pathway via stabilization of $\beta$-catenin, leading to increased susceptibility to CIN (Aoki et al. 2007, Dikovskaya et al. 2007, Caldwell \& Kaplan 2009, Pino \& Chung 2010). While these experimental findings as well as observations of elevated CIN in low-grade dysplastic polyps of FAP patients (Cardoso et al. 2006) and in murine intestinal adenomas with germline mutations in $A P C$ (Alberici et al. 2007, Aoki et al. 2007) provide support for constitutional depletion of APC in predisposition to CIN (Aoki \& Taketo 2007, Pino \& Chung 2010), there have been contradicting findings that argued otherwise (Haigis et al. 2002, Sieber et al. 2002, Aoki \& Taketo 2007). Further investigations demonstrating increased $\mathrm{CIN}$ in cancer patients with germline APC mutations are warranted to unequivocally assign the contribution of APC in CIN predisposition.

Other proteins have also been suggested to affect chromosome segregation by affecting kinetochoremicrotubule dynamics. For instance, deregulation of MAD2, another important SAC protein, has been implicated in promoting chromosome mis-segregation via hyperstabilization of kinetochore-microtubule attachments (Holland \& Cleveland 2012). Transgenic mice overexpressing MAD2 demonstrate an increase in a variety of neoplasms including hepatoma, hepatocellular carcinoma, lung adenomas, fibrosarcomas and lymphomas, with an accompanying increased frequency of chromosomal breaks and whole chromosome gains and losses (Sotillo et al. 2007). Separately, biallelic loss-offunction mutations in CEP57, a kinetochore-microtubule network-binding protein, were detected by whole exome sequencing of a family with MVA syndrome (Snape et al. 2011). It was recently revealed that CEP57 is a kinetochore component and plays a role in SAC signaling (Zhou et al. 2016). CEP57 was shown to localize to kinetochore via its interaction with the kinetochore-microtubule protein MIS12 and activates the SAC by recruitment of MAD1MAD2 components of MCC to the kinetochore. CEP57depleted cells demonstrated attenuated SAC signaling, resulting in premature entry into anaphase that led to an increased rate of chromosome lagging (Zhou et al. 2016). The authors also showed that CEP57 facilitates SAC silencing through competition of microtubule-binding activity with MAD1 (Zhou et al. 2016), suggesting that constitutional aneuploidy associated with CEP57 loss is potentially contributed by increased chromosomal mis-segregation due to impaired CEP57 regulation of kinetochore-microtubule attachments via MAD1-MAD2. Interestingly, loss of function of SNF5, a core component of the SWI/SNF chromatin remodeling complex, has been found to impair SAC signaling and promote chromosome mis-segregation in part by causing overexpression of MAD2 (Vries et al. 2005). SNF is encoded by SMARCB1, which is a known cancer predisposition gene for malignant rhabdoid tumors and familial schwannomatosis (Hulsebos et al. 2007, Eaton et al. 2011). It was observed that SNF5deficient malignant rhabdoid tumor cells exhibiting chromosomal instability in the form of polyploidy had overexpressed MAD2, which could be rescued by the introduction of wild-type SNF5, thus implying the indirect influence of SNF5 on SAC (Vries et al. 2005). However, these observations were contradicted by a separate study that demonstrated most SNF5-deficient tumor cells were genomically stable and the mechanism leading to malignancy was through epigenetic changes, not genomic instability (McKenna et al. 2008). While it remains to be determined whether loss of SNF5 contributes to CIN, the collective evidences indicate the many possible constitutional defects for which SAC and kinetochore-microtubule dynamics may be directly or indirectly affected.

The presence of extra centrosomes has also been shown to increase the incidence of merotelic kinetochore-microtubule attachments by promoting transient multipolar spindles and an association with chromosome lagging during anaphase (Ganem et al. 2009, Thompson et al. 2010). Abnormal numbers of centrosomes have been observed in cells derived from PCS syndrome patients harboring severe CIN (Izumi et al. 2009). Lymphoblastoid cell lines (LCLs) and fibroblasts of these PCS patients with BUB1B germline mutations and constitutional BUBR1 deficiency demonstrated deregulation in the centrosome duplication machinery resulting in centrosome amplification (Izumi et al. 2009). Using BUBR1 deletion mutants, it was shown that

Published by Bioscientifica Ltd 
BUBR1 negatively regulates the kinase activity of PLK1, which is involved in centrosome reduplication during interphase (Izumi et al. 2009). Therefore, it appears that apart from SAC signaling, germline mutations in $B U B 1 B$ may affect kinetochore-microtubule attachments through interference in regulation of centrosome duplication, ultimately increasing the likelihood of chromosome mis-segregation. In turn, a higher frequency of chromosome mis-segregation increases the chances of generating micronuclei containing whole chromosome or chromosome fragments, which can lead to defective or asynchronous DNA replication and accumulation of further DNA damage, such as chromosome breaks and damaging mutations, resulting in chromothripsis and potentially predisposing to cancer (Crasta et al. 2012, Zhang et al. 2015). Chromothripsis is characterized by small-scale DNA copy number changes and extensive intrachromosomal rearrangements confined to single chromosome or chromosome arm and has been featured in human cancers (Crasta et al. 2012, Zhang et al. 2015). Germline chromothripsis is associated with congenital abnormalities as well as developmental disorders (de Pagter et al. 2015, Anderson et al. 2016, Bertelsen et al. 2016). Parent-to-child transmission of chromothripsis has been associated with de novo complex copy number changes and severe congenital abnormalities in the child (de Pagter et al. 2015), suggesting that germline chromothripsis may lead to further CIN and potentially, predisposition to cancers.

\section{Germline mutations affecting DNA damage response}

While germline mutations in SAC or kinetochoremicrotubule dynamics have been demonstrated to promote chromosome mis-segregation leading to CIN, it has been noted in many studies that these germline mutations alone may not be sufficient for tumorigenesis (Bakhoum \& Compton 2012). Additional genetic alterations, affecting genes governing cell cycle regulation or DNA damage response (DDR) such as TP53, CDKN2A, $A T M$ and BRCA1/2 commonly precede tumor formation (Bakhoum \& Compton 2012).

The human cell has evolved a suite of mechanisms to protect the integrity of the genome from the plethora of DNA damages induced by both exogenous and endogenous insults. This includes the DNA damage repair system, which can be broadly viewed as four major pathways: base-excision repair (BER), nucleotide-excision repair (NER), double-strand DNA breaks (DSBs) repair and mismatch repair (MMR) (Hoeijmakers 2001). The interaction and complexities of these mechanisms have been comprehensively reviewed elsewhere (Hoeijmakers 2001, Jackson \& Bartek 2009, Dexheimer 2013). In general, germline alterations in any of these pathways predispose to an elevated risk of cancer. Many human cancer predisposition syndromes such as Fanconi anemia, ataxia-telangiectasia, Nijmegen breakage syndrome, Bloom syndrome and HNPCC are associated with inherited mutations affecting genes involved in these DDR pathways (Hoeijmakers 2001). As it is beyond the scope of this review to address all the syndromes related to defective DDR pathways, we will discuss those with inherited defects in DSBs repair, which are more commonly associated with chromosomal aberrations leading to CIN (Table 1).

DSBs are highly cytotoxic DNA lesions especially during replication because broken chromosomes that are carried through mitosis lead to inaccurate and unbalanced chromosomal segregation between daughter cells, which can propagate the genomic instability (van Gent et al. 2001). The cell has evolved DNA damage signaling and repair response to efficiently trigger cell cycle arrest and repair the lesions before resuming to normal cellular activities (van Gent et al. 2001, Shiloh 2003, Jackson \& Bartek 2009). There are two main modes of DSB repair: homologous recombination (HR) and non-homologous end joining (NHEJ) (Hoeijmakers 2001, Dexheimer 2013). While differing in the mechanisms and fidelity of repair, both HR and NHEJ essentially are in place to fix broken DNA duplex, which can arise from exogenous sources such as ionizing radiation and chemical agents or endogenously from stalled or collapsed DNA replication forks (Chapman et al. 2012, Dexheimer 2013).

The proper function of DSBs repair pathway is important for maintaining genome stability. Inherited defects in key players of this pathway are frequently associated with chromosomal instability and a predisposition to cancer (Hoeijmakers 2001). For instance, germline mutations in BRCA1 and BRCA2 are known to strongly predispose to hereditary breast and ovarian cancers as well as Fanconi anemia, a rare recessive disorder characterized by bone marrow failure, chromosomal instability and early-onset cancer (Hoeijmakers 2001, Garber \& Offit 2005, Ceccaldi et al. 2016). Both BRCA1 and BRCA2 play critical roles in stabilizing the replication fork and mediating the HR process during DSBs repair (Ceccaldi et al. 2016). Mutations in BRCA1 and BRCA2 affecting their expression have been shown to associate with impaired DSBs repair and increased chromosomal

Published by Bioscientifica Ltd 
instability exhibited by broken and deformed chromosomes (Venkitaraman 2004, Deng 2006). It is conceivable how unrepaired DSBs can accumulate due to constitutional BRCA1 or BRCA2 deficiency, gathering more chromosomal abnormalities with each round of replication and ultimately increasing the chances of earlyonset cancer.

Accumulation of DSBs has been observed in cells of patients with cancer syndromes; an example being Werner syndrome (Ariyoshi et al. 2007). Werner syndrome (WS, OMIM 277700) is a rare autosomal recessive disorder associated with premature aging, increased chromosomal instabilities and a higher risk of a variety of malignancies including sarcomas, thyroid carcinomas and melanoma (Goto et al. 1996, Chen \& Oshima 2002, Opresko et al. 2003). WS is associated with germline mutations in WRN (also known as RECQL2), a DNA helicase involved in DSBs repair. The enrichment of DSBs in WS cells suggests that defects in WRN may predispose to chromosomal instabilities via inefficient DSBs repair (Ariyoshi et al. 2007). Another member of the DNA helicase RecQ family, $B L M$ (also known as RECQL3), is associated with Bloom syndrome (OMIM 210900), an autosomal recessive disorder also featuring chromosomal instability and a predisposition to early-onset cancers including leukemia, lymphoma, Wilms tumor and adenocarcinomas (Garber \& Offit 2005, Amor-Guéret 2006, Wu 2016). BLM is also involved in HR and cells with germline defects in $B L M$ showed increased chromosomal breakage and an excess of sister chromatid exchange (SCE), an indicator of DNA damage repair (Wu 2016).

Fanconi anemia (FA, OMIM 227650) is a rare autosomal recessive disorder characterized by bone marrow failure, congenital growth abnormalities, hypersensitivity to interstrand crosslink-inducing agents, chromosomal instability and a predisposition to cancers, especially leukemia, head and neck cancers as well as breast and ovarian cancers (Garber \& Offit 2005, Bogliolo \& Surrallés 2015). There are as many as 19 genes associated with the FA pathway, a subset of the DSBs repair system (Ceccaldi et al. 2016). It has been shown in FA patientderived cells that a combination of chromosome missegregation and unrepaired interphase DNA damage leads to chromosomal instability in FA (Cerabona et al. 2014). Individuals with heterozygous germline mutations in FA genes register a higher mean index of DEB-induced chromosome breakage than controls, suggesting that compromised DNA repair efficiency due to inherited defects in FA pathway may possibly heighten the vulnerability to genomic instability (Mathew 2006).
Before DSBs can be repaired, the DNA damage signal has to trigger the activation of the repair mechanism. One of the principal sensors and signal transducer of DSBs is ATM, a kinase that responds to DSBs by coordinating a suite of DNA damage response via phosphorylation of a wide range of target substrates (Shiloh 2003). Germline mutations in ATM are associated with ataxia-telangiectasia (A-T, OMIM 208900), an autosomal recessive condition characterized by ataxia i.e. progressive loss of muscular coordination, telangiectasia i.e. dilated blood vessels in the skin, immunodeficiencies, chromosomal breakages, as well as predisposition to malignancies including leukemia, lymphoma, sarcoma, ovarian, breast and gastric cancers (Shiloh 2003, Garber \& Offit 2005, Wu 2016). The critical role of $A T M$ in facilitating various response mechanisms to DSBs ranging from cell cycle arrest to activating the cascade of HR proteins required for DSBs repair implies that germline mutations in ATM resulting in ATM deficiency impairs healing of broken DNA with concomitant deregulation of cell cycle arrest, thereby increasing the risk of fragmented chromosomes being propagated over subsequent mitoses. One of the substrates of ATM is NBN, a component of the MRE11/RAD50/NBN (MRN) protein complex important for DSBs sensing, repair as well as DSB-induced cell cycle signaling (Shiloh 2003, Lamarche et al. 2010). Germline mutations in NBN are associated with Nijmegen breakage syndrome (NBS), which unsurprisingly is phenotypically similar to A-T, given the substantial overlap in the molecular pathway (Shiloh 2003). NBS patients are predisposed to multiple malignancies including early-onset lymphoma, brain tumors as well as rhabdomyosarcoma, and their cells show a higher incidence of chromosomal instability (Garber \& Offit 2005, Wu 2016).

\section{Clinical significance}

Because CIN is a common feature of cancer, it makes a compelling target for anti-cancer therapy. While numerous studies demonstrated that CIN may promote tumorigenesis, predominantly via the functional loss of key players governing chromosomal stability, it has also been shown that CIN beyond tolerable levels in fact results in cell death and tumor suppression (Weaver et al. 2007, Holland \& Cleveland 2009, Thompson et al. 2010, Holland \& Cleveland 2012).

In vitro and in vivo observations from several studies have demonstrated that low levels of CIN may promote tumor initiation but at elevated levels, CIN may actually be protective and inhibit tumorigenesis (Rao et al. 2005,

Published by Bioscientifica Ltd. 
Weaver et al. 2007, Silk etal. 2013, Zasadil et al. 2016). It was noticed in an earlier investigation that BubR1+/- ApcMin/+ compound mutant mice, which exhibited increased karyotypic instability, were found to have increased formation of colonic polyps but at the same time, a notable decrease in small intestine adenomas (Rao et al. 2005). In a landmark study, increased CIN associated with reduced CENP-E expression in CENP-E $\mathrm{E}^{+/}$mice was found to promote tumor development in lymphocytes and lungs, where aneuploidy is inherently low, but inhibited tumor growth in the liver, which has inherently higher levels of aneuploidy (Weaver et al. 2007). More recently, it has been demonstrated that the increased rate of chromosome mis-segregation in compound mutant $\mathrm{Apc}^{\mathrm{Min} /+} \mathrm{CENP}_{-\mathrm{E}}^{+/-}$mice is associated with greater cell death and suppression of tumor progression compared to singly mutant $\mathrm{Apc}^{\mathrm{Min} /+}$ and CENP-E ${ }^{+/-}$mice (Zasadil et al. 2016). These observations collectively suggest elevating CIN as a potential chemotherapeutic strategy in a genetically sensitized background. Indeed, a preliminary study using cells with transiently induced CIN (through silencing of SKA3 and MIS18) showed that it is possible to elevate chromosome mis-segregation upon application of the chemotherapeutic drugs taxol and gemcitabine at doses below their IC $_{50}$ (Lee et al. 2016). Further studies and trials would be necessary to validate the efficacy and efficiency of this approach to cancer treatment, but this possibility opens up therapeutic avenues involving the selective elevation of CIN to lethal levels in patients harboring constitutional CIN through the use of less toxic doses of chemotherapy drugs to promote tumor cell death (Lee et al. 2016).

A strategy to cancer therapy that has been gaining traction in recent years is synthetic lethality (Kaelin 2005, Helleday et al. 2008, Curtin 2012, Gavande et al. 2016). Synthetic lethality is the circumstance in which genetic defects in two different genes or pathways together leads to cell death but when either occurred alone, is compatible with viability (Kaelin 2005, Curtin 2012). One of the successful examples to date is the application of poly(ADP-ribose) polymerase inhibitors (PARPis) to specifically kill BRCA1/2-deficient tumors (Bryant et al. 2005, Curtin 2012, Gavande et al. 2016, Lim \& Ngeow 2016). PARP is a nuclear protein important for recognizing DNA damage and repairing DNA singlestrand breaks (SSBs) (Gavande et al. 2016). It is proposed that inhibition of PARP prevents its release from damaged DNA, resulting in the accumulation of unrepaired SSBs that would give rise to DSBs, which is typically resolved by HR repair (Curtin 2012, Gavande et al. 2016). The lack of a functional HR repair mechanism in BRCA1/2deficient cells renders vulnerability to cell death upon PARPi administration (Bryant et al. 2005). Clinical trials using PARPi, namely olaparib and rucaparib, in BRCAassociated cancers such as breast and ovarian cancers have been encouraging (Kaufman et al. 2015, Konecny \& Kristeleit 2016). In particular, ovarian cancer patients with germline BRCA mutations or BRCA-like $\mathrm{LOH}$ demonstrated a more favorable response to rucaparib compared to those with wild-type BRCA (Konecny \& Kristeleit 2016). The promising progress of PARPi trials in HR-deficient cancers suggests that synthetic lethality may be a feasible therapeutic strategy for cancer patients with germline mutations in DNA damage response due to the sensitization of tumor to the catastrophic consequences of failed DNA damage repair. In addition to monotherapy, it is possible to combine this approach of synthetic lethality with microtubule poisons to potentially elevate CIN and enhance cytotoxicity on tumor cells. For instance, it has been shown that ATM deficiency and PARPi are synthetic lethal, and the combination of olaparib with paclitaxel has been observed to elicit an improved response in cancer patients with reduced ATM expression (Dréan et al. 2016). Thus, it is possible that patients with germline mutations in ATM may benefit from the synergistic effect of a combinatorial $\mathrm{PARPi} /$ microtubule poison therapy against a genetically sensitized background. The encouraging success of the synthetic lethal approach demonstrated by PARPi and BRCA deficiency opens up opportunities for considering other targets in DNA damage response beyond PARPi and BRCA deficiency. Indeed, synthetic lethality has been shown for ATM inhibition and FA pathway deficiency (Hosoya \& Miyagawa 2014), suggesting the potential of applying ATM inhibitors in FA patients with germline mutations in FA genes.

Collectively, these emerging evidences demonstrate that it is possible to exploit the vulnerabilities conferred by germline mutations predisposing to CIN for exploration of novel cancer treatment strategies (Fig. 2).

\section{Future directions}

Over the last decade, the extensive cataloging and dissection of patterns in base substitution somatic mutations on various cancer types by multiple studies have uncovered strong associations of distinct patterns with specific tumor types (Greenman et al. 2007, 
Alexandrov et al. 2013, Alexandrov \& Stratton 2014). Different tumor types (and subtypes) have unique patterns, termed somatic mutational signatures, from which the etiology of mutagenesis may be inferred (Alexandrov et al. 2013, Helleday et al. 2014, Forbes et al. 2017). It has been shown that defects in the maintenance of genomic DNA integrity are strongly associated with a distinctive few of these reported mutational signatures. Importantly, tumors with HR deficiency such as breast, ovarian and pancreatic tumors with germline/somatic mutations in BRCA1 or BRCA2 are shown to harbor mutational signature 3 (Alexandrov et al. 2013, Nik-Zainal et al. 2016, $15)$, whereas signatures 6,20 and 26 are frequently found in microsatellite unstable tumors, such as colorectal and uterine cancers with defective DNA mismatch repair (Alexandrov et al. 2013, Forbes et al. 2017). Moreover, as seen in breast cancer, it was possible to distinguish tumors with germline mutations in susceptibility genes through differences in the relative contributions of each component signature (Nik-Zainal et al. 2012, Alexandrov \& Stratton 2014). In addition to base substitution signatures, signatures of rearrangement mutational processes such as deletions, inversions, tandem duplications and interchromosomal translocations have recently been reported for breast cancer (Nik-Zainal et al. 2016). In this study, the authors alluded to an improved diagnostic utility for HR deficiency in breast tumors by merging base substitution and rearrangement mutational signatures for a more comprehensive and characteristic profile (Nik-Zainal et al. 2016).

Taken together, it is conceivable that tumors with germline defects predisposing to chromosomal instability may exhibit a distinctive somatic mutational signature. A recent study, which correlated the mutational signature 18 with pathogenic germline mutations in MUTYH in colorectal cancer, demonstrated the potential of using this

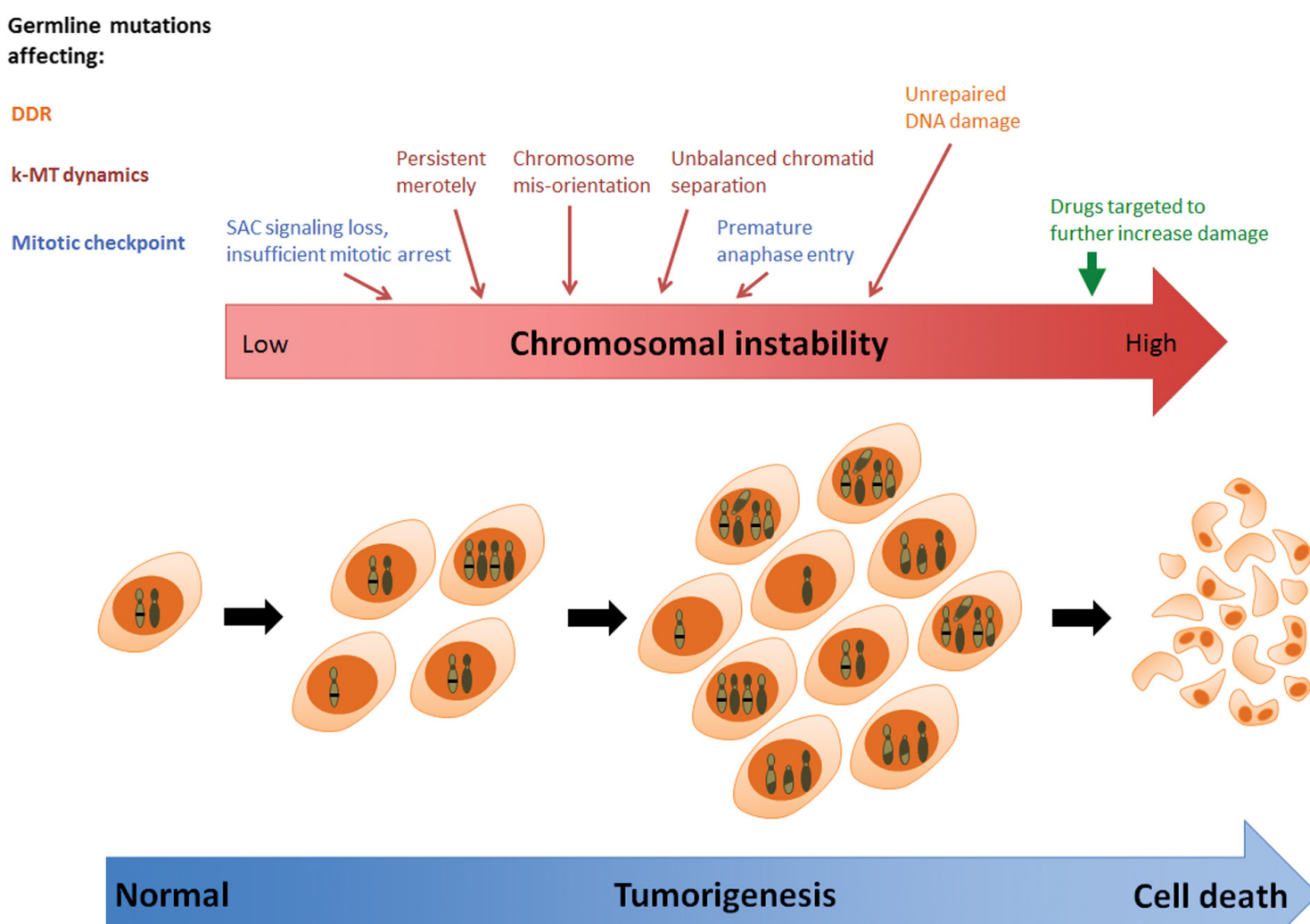

\section{Figure 2}

CIN can promote both tumor progression and tumor suppression. Germline mutations affecting the mitotic checkpoint, kinetochore-microtubule (k-MT) dynamics and DNA damage response can predispose to CIN as a result of insufficient delays in mitotic arrest to repair aberrations such as merotelic attachments, chromosomal mis-orientations and DNA damage. Consequently, unbalanced and broken DNA persists over subsequent mitoses, generating daughter cells with ever-increasing levels of chromosomal instability. Further loss of critical tumor suppressors (or amplification of oncogenes) coupled with selective growth advantage and clonal expansion leads to tumorigenesis. However, CIN beyond tolerable levels is non-viable and often leads to cell death. By tipping the balance of CIN in the cell, such as using targeted drugs that is synthetic lethal to the existing germline defect, it is possible to exploit this vulnerability to promote tumor suppression as a therapeutic strategy. 
mutational signature as a biomarker to identify MUTYH germline mutations in a sample set of adrenocortical carcinomas (Pilati et al. 2017). Is there a somatic base substitution or rearrangement mutational signature that we can identify to correlate CIN with inherent genetic alterations, such as germline BUB1B mutations? Comparison of mutational signatures across various CIN tumors may elucidate distinct patterns suggestive of defective mechanisms in DNA maintenance to help us address this question. It would also be interesting to find out whether there is a mutational signature characteristic of a specific syndrome, for instance, MVA. In addition, can we find a common mutational signature in CIN tumors with good response to specific therapies, such as PARPi discussed above? If found, the mutational signature(s) may represent a potential biomarker that not only facilitates diagnosis and prognosis, but will also have big implications in molding our therapeutic as well as patient management strategies. By leveraging on capabilities of next-generation sequencing on tumor profiling and germline testing, we would be able to integrate somatic and germline information in a concerted effort to comprehensively understand the germline contribution to CIN and infer potential therapeutic outcomes in a step toward more effective personalized treatment for cancer patients (Ngeow \& Eng 2016).

\section{Summary}

Constitutional CIN is a known hallmark of cancer. While it remains a continuing topic for debate whether CIN precedes tumor initiation or is merely a secondary outcome of tumorigenesis, there are substantial evidences supporting that germline mutation in key genes central to mitosis can lead to impairment of the mechanisms governing faithful chromosomal segregation, thus predisposing cells to CIN. The association of germline BUB1B mutations with early-onset malignancies in patients of MVA and PCS syndromes, in which constitutional CIN is a feature, is an example of inherited mutations predisposing to CIN can elevate one's lifetime risk of developing cancer. As new candidate genes associated with constitutional CIN are being discovered and experimental evidences accumulate to validate their mechanisms of action, we will also stand to gain new insights on how these genes may predispose to cancer. This would not only expand our understanding of CIN in cancer predisposition but integration of these continuously evolving insights into our patient management strategies will help us further improve our clinical care.
Declaration of interest

The authors declare that there is no conflict of interest that could be perceived as prejudicing the impartiality of this review.

\section{Funding}

This work did not receive any specific grant from any funding agency in the public, commercial, or not-for-profit sector.

\section{References}

Alberici P, de Pater E, Cardoso J, Bevelander M, Molenaar L, Jonkers J \& Fodde R 2007 Aneuploidy arises at early stages of Apc-driven intestinal tumorigenesis and pinpoints conserved chromosomal loci of allelic imbalance between mouse and human. American Journal of Pathology 170 377-387. (doi:10.2353/ ajpath.2007.060853)

Alexandrov LB \& Stratton MR 2014 Mutational signatures: the patterns of somatic mutations hidden in cancer genomes. Current Opinion in Genetics and Development 24 52-60. (doi:10.1016/j.gde.2013.11.014)

Alexandrov LB, Nik-Zainal S, Wedge DC, Aparicio SAJR, Behjati S, Biankin AV, Bignell GR, Bolli N, Borg A, Børresen-Dale A-L, et al. 2013 Signatures of mutational processes in human cancer. Nature 500 415-421. (doi:10.1038/nature12477)

Amor-Guéret M 2006 Bloom syndrome, genomic instability and cancer: the SOS-like hypothesis. Cancer Letters 236 1-12. (doi:10.1016/j. canlet.2005.04.023)

Anderson SE, Kamath A, Pilz DT \& Morgan SM 2016 A rare example of germ-line chromothripsis resulting in large genomic imbalance. Clinical Dysmorphology 25 58-62. (doi:10.1097/ MCD.0000000000000113)

Aoki K \& Taketo MM 2007 Adenomatous polyposis coli (APC): a multifunctional tumor suppressor gene. Journal of Cell Science $\mathbf{1 2 0}$ 3327-3335. (doi:10.1242/jcs.03485)

Aoki K, Aoki M, Sugai M, Harada N, Miyoshi H, Tsukamoto T, Mizoshita T, Tatematsu M, Seno H, Chiba T, et al. 2007 Chromosomal instability by beta-catenin/TCF transcription in APC or beta-catenin mutant cells. Oncogene 26 3511-3520. (doi:10.1038/sj.onc.1210141)

Ariyoshi K, Suzuki K, Goto M, Watanabe M \& Kodama S 2007 Increased chromosome instability and accumulation of DNA double-strand breaks in Werner syndrome cells. Journal of Radiation Research $\mathbf{4 8}$ 219-231. (doi:10.1269/jrr.07017)

Bahmanyar S, Nelson WJ \& Barth AIM 2009 Role of APC and its binding partners in regulating microtubules in mitosis. Advances in Experimental Medicine and Biology 656 65-74. (doi:10.1007/978-14419-1145-2_6)

Bakhoum SF \& Compton DA 2012 Chromosomal instability and cancer: a complex relationship with therapeutic potential. Journal of Clinical Investigation 122 1138-1143. (doi:10.1172/JCI59954)

Bakhoum SF, Genovese G \& Compton DA 2009 Deviant kinetochore microtubule dynamics underlie chromosomal instability. Current Biology 19 1937-1942. (doi:10.1016/j.cub.2009.09.055)

Bertelsen B, Nazaryan-Petersen L, Sun W, Mehrjouy MM, Xie G, Chen W, Hjermind LE, Taschner PEM \& Tümer Z 2016 A germline chromothripsis event stably segregating in 11 individuals through three generations. Genetics in Medicine 18 494-500. (doi:10.1038/ $\operatorname{gim} .2015 .112)$

Bogliolo M \& Surrallés J 2015 Fanconi anemia: a model disease for studies on human genetics and advanced therapeutics. Current Opinion in Genetics and Development 33 32-40. (doi:10.1016/j.gde.2015.07.002)

Boland CR \& Goel A 2010 Microsatellite instability in colorectal cancer. Gastroenterology 138 2073.e3-2087.e3. (doi:10.1053/j. gastro.2009.12.064)

Published by Bioscientifica Ltd. 
Bryant HE, Schultz N, Thomas HD, Parker KM, Flower D, Lopez E, Kyle S, Meuth M, Curtin NJ \& Helleday T 2005 Specific killing of BRCA2deficient tumours with inhibitors of poly(ADP-ribose) polymerase. Nature 434 913-917. (doi:10.1038/nature03443)

Caldwell CM \& Kaplan KB 2009 The role of APC in mitosis and in chromosome instability. Advances in Experimental Medicine and Biology 656 51-64. (doi:10.1007/978-1-4419-1145-2_5)

Cardoso J, Molenaar L, de Menezes RX, van Leerdam M, Rosenberg C, Möslein G, Sampson J, Morreau H, Boer JM \& Fodde R 2006 Chromosomal instability in MYH- and APC-mutant adenomatous polyps. Cancer Research 66 2514-2519. (doi:10.1158/0008-5472.CAN05-2407)

Carmena M, Ruchaud S \& Earnshaw WC 2009 Making the Auroras glow: regulation of Aurora A and B kinase function by interacting proteins. Current Opinion in Cell Biology 21 796-805. (doi:10.1016/j. ceb.2009.09.008)

Ceccaldi R, Sarangi P \& D'Andrea AD 2016 The Fanconi anaemia pathway: new players and new functions. Nature Reviews Molecular Cell Biology 17 337-349. (doi:10.1038/nrm.2016.48)

Cerabona D, Sater ZA, Enzor R \& Nalepa G 2014 Genomic instability in fanconi anemia results from a combination of chromosome missegregation in mitosis and unresolved interphase DNA damage. Blood 124 357-357.

Chan YW, Jeyaprakash AA, Nigg EA \& Santamaria A 2012 Aurora B controls kinetochore-microtubule attachments by inhibiting Ska complex-KMN network interaction. Journal of Cell Biology 196 563-571. (doi:10.1083/jcb.201109001)

Chapman JR, Taylor MRG \& Boulton SJ 2012 Playing the end game: DNA double-strand break repair pathway choice. Molecular Cell 47 497-510. (doi:10.1016/j.molcel.2012.07.029)

Chen L \& Oshima J 2002 Werner syndrome. Journal of Biomedicine and Biotechnology 2 46-54. (doi:10.1155/S1110724302201011)

Cimini D, Wan X, Hirel CB \& Salmon ED 2006 Aurora kinase promotes turnover of kinetochore microtubules to reduce chromosome segregation errors. Current Biology 16 1711-1718. (doi:10.1016/j. cub.2006.07.022)

Crasta K, Ganem NJ, Dagher R, Lantermann AB, Ivanova EV, Pan Y, Nezi L, Protopopov A, Chowdhury D \& Pellman D 2012 DNA breaks and chromosome pulverization from errors in mitosis. Nature 482 53-58. (doi:10.1038/nature10802)

Curtin NJ 2012 DNA repair dysregulation from cancer driver to therapeutic target. Nature Reviews Cancer 12 801-817. (doi:10.1038/ nrc3399)

Deng C-X 2006 BRCA1: cell cycle checkpoint, genetic instability, DNA damage response and cancer evolution. Nucleic Acids Research $\mathbf{3 4}$ 1416-1426. (doi:10.1093/nar/gkl010)

de Pagter MS, van Roosmalen MJ, Baas AF, Renkens I, Duran KJ, van Binsbergen E, Tavakoli-Yaraki M, Hochstenbach R, van der Veken LT, Cuppen E, et al. 2015 Chromothripsis in healthy individuals affects multiple protein-coding genes and can result in severe congenital abnormalities in offspring. American Journal of Human Genetics 96 651-656. (doi:10.1016/j.ajhg.2015.02.005)

de Voer RM, Hoogerbrugge N \& Kuiper RP 2011 Spindle-assembly checkpoint and gastrointestinal cancer. New England Journal of Medicine 364 1279-1280. (doi:10.1056/NEJMc1101053)

de Voer RM, Geurts van Kessel A, Weren RDA, Ligtenberg MJL, Smeets D, Fu L, Vreede L, Kamping EJ, Verwiel ETP, Hahn M-M, et al. 2013 Germline mutations in the spindle assembly checkpoint genes BUB1 and BUB3 are risk factors for colorectal cancer. Gastroenterology $\mathbf{1 4 5}$ 544-547. (doi:10.1053/j.gastro.2013.06.001)

Dexheimer TS 2013 DNA repair pathways and mechanisms. In DNA Repair of Cancer Stem Cells, pp 19-32. Eds LA Mathews, SM Cabarcas \& EM Hurt. Netherlands: Springer. (doi:10.1007/978-94-0074590-2_2)

Dikovskaya D, Schiffmann D, Newton IP, Oakley A, Kroboth K, Sansom O, Jamieson TJ, Meniel V, Clarke A \& Näthke IS 2007 Loss of APC induces polyploidy as a result of a combination of defects in mitosis and apoptosis. Journal of Cell Biology 176 183-195. (doi:10.1083/ jcb.200610099)

Draviam VM, Shapiro I, Aldridge B \& Sorger PK 2006 Misorientation and reduced stretching of aligned sister kinetochores promote chromosome missegregation in EB1- or APC-depleted cells. EMBO Journal 25 2814-2827. (doi:10.1038/sj.emboj.7601168)

Dréan A, Lord CJ \& Ashworth A 2016 PARP inhibitor combination therapy. Critical Reviews in Oncology/Hematology 108 73-85. (doi:10.1016/j.critrevonc.2016.10.010)

Eaton KW, Tooke LS, Wainwright LM, Judkins AR \& Biegel JA 2011 Spectrum of SMARCB1/INI1 mutations in familial and sporadic rhabdoid tumors. Pediatric Blood and Cancer 56 7-15. (doi:10.1002/ pbc.22831)

Fernández-Miranda G, Trakala M, Martín J, Escobar B, González A, Ghyselinck NB, Ortega S, Cañamero M, Castro IP de \& Malumbres M 2011 Genetic disruption of aurora B uncovers an essential role for aurora C during early mammalian development. Development 138 2661-2672. (doi:10.1242/dev.066381)

Fodde R, Kuipers J, Rosenberg C, Smits R, Kielman M, Gaspar C, van Es JH, Breukel C, Wiegant J, Giles RH, et al. 2001 Mutations in the APC tumour suppressor gene cause chromosomal instability. Nature Cell Biology 3 433-438. (doi:10.1038/35070129)

Foley EA \& Kapoor TM 2013 Microtubule attachment and spindle assembly checkpoint signalling at the kinetochore. Nature Reviews Molecular Cell Biology 14 25-37. (doi:10.1038/nrm3494)

Forbes SA, Beare D, Boutselakis H, Bamford S, Bindal N, Tate J, Cole CG, Ward S, Dawson E, Ponting L, et al. 2017 COSMIC: somatic cancer genetics at high-resolution. Nucleic Acids Research 45 D777-D783. (doi:10.1093/nar/gkw1121)

Ganem NJ, Godinho SA \& Pellman D 2009 A mechanism linking extra centrosomes to chromosomal instability. Nature 460 278-282. (doi:10.1038/nature08136)

Garber JE \& Offit K 2005 Hereditary cancer predisposition syndromes. Journal of Clinical Oncology 23 276-292. (doi:10.1200/ JCO.2005.10.042)

García-Castillo H, Vásquez-Velásquez AI, Rivera H \& Barros-Núñez P 2008 Clinical and genetic heterogeneity in patients with mosaic variegated aneuploidy: delineation of clinical subtypes. American Journal of Medical Genetics: Part A 146A 1687-1695. (doi:10.1002/ajmg.a.32315)

Gavande NS, VanderVere-Carozza PS, Hinshaw HD, Jalal SI, Sears CR, Pawelczak KS \& Turchi JJ 2016 DNA repair targeted therapy: the past or future of cancer treatment? Pharmacology and Therapeutics 160 65-83. (doi:10.1016/j.pharmthera.2016.02.003)

Giam M \& Rancati G 2015 Aneuploidy and chromosomal instability in cancer: a jackpot to chaos. Cell Division 10 3. (doi:10.1186/s13008015-0009-7)

Goto M, Miller RW, Ishikawa Y \& Sugano H 1996 Excess of rare cancers in Werner syndrome (adult progeria). Cancer Epidemiology, Biomarkers and Prevention 5 239-246.

Greenman C, Stephens P, Smith R, Dalgliesh GL, Hunter C, Bignell G, Davies H, Teague J, Butler A, Stevens C, et al. 2007 Patterns of somatic mutation in human cancer genomes. Nature 446 153-158. (doi:10.1038/nature05610)

Gregan J, Polakova S, Zhang L, Tolić-Nørrelykke IM \& Cimini D 2011 Merotelic kinetochore attachment: causes and effects. Trends in Cell Biology 21 374-381. (doi:10.1016/j.tcb.2011.01.003)

Hahn M-M, Vreede L, Bemelmans SASA, van der Looij E, van Kessel AG, Schackert HK, Ligtenberg MJL, Hoogerbrugge N, Kuiper RP \& de Voer RM 2016 Prevalence of germline mutations in the spindle assembly checkpoint gene BUB1B in individuals with early-onset colorectal cancer. Genes, Chromosomes and Cancer 55 855-863. (doi:10.1002/gcc.22385)

Haigis KM, Caya JG, Reichelderfer M \& Dove WF 2002 Intestinal adenomas can develop with a stable karyotype and stable microsatellites. PNAS 99 8927-8931. (doi:10.1073/pnas.132275099) 
Hanks S, Coleman K, Reid S, Plaja A, Firth H, Fitzpatrick D, Kidd A, Méhes K, Nash R, Robin N, et al. 2004 Constitutional aneuploidy and cancer predisposition caused by biallelic mutations in BUB1B. Nature Genetics 36 1159-1161. (doi:10.1038/ng1449)

Helleday T, Petermann E, Lundin C, Hodgson B \& Sharma RA 2008 DNA repair pathways as targets for cancer therapy. Nature Reviews Cancer $\mathbf{8}$ 193-204. (doi:10.1038/nrc2342)

Helleday T, Eshtad S \& Nik-Zainal S 2014 Mechanisms underlying mutational signatures in human cancers. Nature Reviews Genetics $\mathbf{1 5}$ 585-598. (doi:10.1038/nrg3729)

Hoeijmakers JHJ 2001 Genome maintenance mechanisms for preventing cancer. Nature 411 366-374. (doi:10.1038/35077232)

Holland AJ \& Cleveland DW 2009 Boveri revisited: chromosomal instability, aneuploidy and tumorigenesis. Nature Reviews Molecular Cell Biology 10 478-487. (doi:10.1038/nrm2718)

Holland AJ \& Cleveland DW 2012 Losing balance: the origin and impact of aneuploidy in cancer. EMBO Reports 13 501-514. (doi:10.1038/embor.2012.55)

Hosoya N \& Miyagawa K 2014 Targeting DNA damage response in cancer therapy. Cancer Science 105 370-388. (doi:10.1111/cas.12366)

Hulsebos TJM, Plomp AS, Wolterman RA, Robanus-Maandag EC, Baas F \& Wesseling P 2007 Germline mutation of INI1/SMARCB1 in familial schwannomatosis. American Journal of Human Genetics $\mathbf{8 0}$ 805-810. (doi:10.1086/513207)

Izumi H, Matsumoto Y, Ikeuchi T, Saya H, Kajii T \& Matsuura S 2009 BubR1 localizes to centrosomes and suppresses centrosome amplification via regulating Plk1 activity in interphase cells. Oncogene 28 2806-2820. (doi:10.1038/onc.2009.141)

Jackson SP \& Bartek J 2009 The DNA-damage response in human biology and disease. Nature 461 1071-1078. (doi:10.1038/nature08467)

Kaelin WG 2005 The concept of synthetic lethality in the context of anticancer therapy. Nature Reviews Cancer 5 689-698. (doi:10.1038/ nrc1691)

Kaplan KB, Burds AA, Swedlow JR, Bekir SS, Sorger PK \& Näthke IS $2001 \mathrm{~A}$ role for the Adenomatous polyposis coli protein in chromosome segregation. Nature Cell Biology 3 429-432. (doi:10.1038/35070123)

Kaufman B, Shapira-Frommer R, Schmutzler RK, Audeh MW, Friedlander M, Balmaña J, Mitchell G, Fried G, Stemmer SM, Hubert A, et al. 2015 Olaparib monotherapy in patients with advanced cancer and a germline BRCA1/2 mutation. Journal of Clinical Oncology 33 244-250. (doi:10.1200/JCO.2014.56.2728)

Klotz DM, Nelson SA, Kroboth K, Newton IP, Radulescu S, Ridgway RA, Sansom OJ, Appleton PL \& Näthke IS 2012 The microtubule poison vinorelbine kills cells independently of mitotic arrest and targets cells lacking the APC tumour suppressor more effectively. Journal of Cell Science 125 887-895. (doi:10.1242/jcs.091843)

Knudson AG 1996 Hereditary cancer: two hits revisited. Journal of Cancer Research and Clinical Oncology 122 135-140. (doi:10.1007/BF01366952)

Knudson AG 2001 Two genetic hits (more or less) to cancer. Nature Reviews Cancer 1 157-162. (doi:10.1038/35101031)

Konecny GE \& Kristeleit RS 2016 PARP inhibitors for BRCA1/2-mutated and sporadic ovarian cancer: current practice and future directions. British Journal of Cancer 115 1157-1173. (doi:10.1038/bjc.2016.311)

Kops GJPL, Weaver BAA \& Cleveland DW 2005 On the road to cancer: aneuploidy and the mitotic checkpoint. Nature Reviews Cancer $\mathbf{5}$ 773-785. (doi:10.1038/nrc1714)

Lamarche BJ, Orazio NI \& Weitzman MD 2010 The MRN complex in double-strand break repair and telomere maintenance. FEBS Letters 584 3682-3695. (doi:10.1016/j.febslet.2010.07.029)

Lara-Gonzalez P, Westhorpe FG \& Taylor SS 2012 The spindle assembly checkpoint. Current Biology 22 R966-R980. (doi:10.1016/j. cub.2012.10.006)

Lee H-S, Lee NCO, Kouprina N, Kim J-H, Kagansky A, Bates S, Trepel JB, Pommier Y, Sackett D \& Larionov V 2016 Effects of anticancer drugs on chromosome instability and new clinical implications for tumor-suppressing therapies. Cancer Research 76 902-911. (doi:10.1158/0008-5472.CAN-15-1617)

Lengauer C, Kinzler KW \& Vogelstein B 1998 Genetic instabilities in human cancers. Nature 396 643-649. (doi:10.1038/25292)

Lim D \& Ngeow J 2016 Evaluation of the methods to identify patients who may benefit from PARP inhibitor use. Endocrine-Related Cancer 23 R267-R285. (doi:10.1530/ERC-16-0116)

Mathew CG 2006 Fanconi anaemia genes and susceptibility to cancer. Oncogene 25 5875-5884. (doi:10.1038/sj.onc.1209878)

Matsuura S, Matsumoto Y, Morishima K, Izumi H, Matsumoto H, Ito E, Tsutsui K, Kobayashi J, Tauchi H, Kajiwara Y, et al. 2006 Monoallelic BUB1B mutations and defective mitotic-spindle checkpoint in seven families with premature chromatid separation (PCS) syndrome. American Journal of Medical Genetics: Part A 140 358-367. (doi:10.1002/ajmg.a.31069)

McKenna ES, Sansam CG, Cho Y-J, Greulich H, Evans JA, Thom CS, Moreau LA, Biegel JA, Pomeroy SL \& Roberts CWM 2008 Loss of the epigenetic tumor suppressor SNF5 leads to cancer without genomic instability. Molecular and Cellular Biology 28 6223-6233. (doi:10.1128/MCB.00658-08)

Musacchio A 2015 The molecular biology of spindle assembly checkpoint signaling dynamics. Current Biology 25 R1002-R1018. (doi:10.1016/j.cub.2015.08.051)

Negrini S, Gorgoulis VG \& Halazonetis TD 2010 Genomic instability an evolving hallmark of cancer. Nature Reviews Molecular Cell Biology 11 220-228. (doi:10.1038/nrm2858)

Ngeow J \& Eng C 2016 Precision medicine in heritable cancer: when somatic tumour testing and germline mutations meet. NPJ Genomic Medicine 1 15006. (doi:10.1038/npigenmed.2015.6)

Nik-Zainal S, Alexandrov LB, Wedge DC, Van Loo P, Greenman CD, Raine K, Jones D, Hinton J, Marshall J, Stebbings LA, et al. 2012 Mutational processes molding the genomes of 21 breast cancers. Cell 149 979-993. (doi:10.1016/j.cell.2012.04.024)

Nik-Zainal S, Davies H, Staaf J, Ramakrishna M, Glodzik D, Zou X, Martincorena I, Alexandrov LB, Martin S, Wedge DC, et al. 2016 Landscape of somatic mutations in 560 breast cancer wholegenome sequences. Nature 534 47-54. (doi:10.1038/nature17676)

Ochiai H, Miyamoto T, Kanai A, Hosoba K, Sakuma T, Kudo Y, Asami K, Ogawa A, Watanabe A, Kajii T, et al. 2014 TALEN-mediated singlebase-pair editing identification of an intergenic mutation upstream of BUB1B as causative of PCS (MVA) syndrome. PNAS 111 1461-1466. (doi:10.1073/pnas.1317008111)

Opresko PL, Cheng W-H, von Kobbe C, Harrigan JA \& Bohr VA 2003 Werner syndrome and the function of the Werner protein; what they can teach us about the molecular aging process. Carcinogenesis 24 791-802. (doi:10.1093/carcin/bgg034)

Pikor L, Thu K, Vucic E \& Lam W 2013 The detection and implication of genome instability in cancer. Cancer Metastasis Reviews 32 341-352. (doi:10.1007/s10555-013-9429-5)

Pilati C, Shinde J, Alexandrov LB, Assié G, André T, Hélias-Rodzewicz Z Doucoudray R, Le Corre D, Zucman-Rossi J, Emile J-F, et al. 2017 Mutational signature analysis identifies MUTYH deficiency in colorectal cancers and adrenocortical carcinomas. Journal of Pathology 242 10-15. (doi:10.1002/path.4880)

Pino MS \& Chung DC 2010 The chromosomal instability pathway in colon cancer. Gastroenterology 138 2059-2072. (doi:10.1053/j. gastro.2009.12.065)

Rao CV, Yang Y-M, Swamy MV, Liu T, Fang Y, Mahmood R, JhanwarUniyal M \& Dai W 2005 Colonic tumorigenesis in BubR1+/ApcMin/+ compound mutant mice is linked to premature separation of sister chromatids and enhanced genomic instability. PNAS 102 4365-4370. (doi:10.1073/pnas.0407822102)

Rio Frio T, Lavoie J, Hamel N, Geyer FC, Kushner YB, Novak DJ, Wark L, Capelli C, Reis-Filho JS, Mai S, et al. 2010 Homozygous BUB1B mutation and susceptibility to gastrointestinal neoplasia. New England Journal of Medicine 363 2628-2637. (doi:10.1056/NEJMoa1006565) 
Roberts NJ, Norris AL, Petersen GM, Bondy ML, Brand R, Gallinger S, Kurtz RC, Olson SH, Rustgi AK, Schwartz AG, et al. 2016 Whole genome sequencing defines the genetic heterogeneity of familial pancreatic cancer. Cancer Discovery 6 166-175. (doi:10.1158/2159. 8290.CD-15-0402)

Rusan NM \& Peifer M 2008 Original CIN: reviewing roles for APC in chromosome instability. Journal of Cell Biology 181 719-726. (doi:10.1083/jcb.200802107)

Santaguida S \& Amon A 2015 Short- and long-term effects of chromosome mis-segregation and aneuploidy. Nature Reviews Molecular Cell Biology 16 473-485. (doi:10.1038/nrm4025)

Schuyler SC, Wu Y-F \& Kuan VJ-W 2012 The Mad1-Mad2 balancing act - a damaged spindle checkpoint in chromosome instability and cancer. Journal of Cell Science 125 4197-4206. (doi:10.1242/ jcs.107037)

Schvartzman J-M, Sotillo R \& Benezra R 2010 Mitotic chromosomal instability and cancer: mouse modelling of the human disease. Nature Reviews Cancer 10 102-115. (doi:10.1038/nrc2781)

Shandilya J, Medler KF \& Roberts SGE 2016 Regulation of AURORA B function by mitotic checkpoint protein MAD2. Cell Cycle $\mathbf{1 5}$ 2196-2201. (doi:10.1080/15384101.2016.1200773)

Shiloh Y 2003 ATM and related protein kinases: safeguarding genome integrity. Nature Reviews Cancer 3 155-168. (doi:10.1038/nrc1011)

Sieber OM, Heinimann K, Gorman P, Lamlum H, Crabtree M, Simpson CA, Davies D, Neale K, Hodgson SV, Roylance RR, et al. 2002 Analysis of chromosomal instability in human colorectal adenomas with two mutational hits at APC. PNAS 99 16910-16915. (doi:10.1073/pnas.012679099)

Silk AD, Zasadil LM, Holland AJ, Vitre B, Cleveland DW \& Weaver BA 2013 Chromosome missegregation rate predicts whether aneuploidy will promote or suppress tumors. PNAS 110 E4134-E4141. (doi:10.1073/pnas.1317042110)

Snape K, Hanks S, Ruark E, Barros-Núñez P, Elliott A, Murray A, Lane AH, Shannon N, Callier P, Chitayat D, et al. 2011 Mutations in CEP57 cause mosaic variegated aneuploidy syndrome. Nature Genetics 43 527-529. (doi:10.1038/ng.822)
Sotillo R, Hernando E, Díaz-Rodríguez E, Teruya-Feldstein J, CordónCardo C, Lowe SW \& Benezra R 2007 Mad2 overexpression promotes aneuploidy and tumorigenesis in mice. Cancer Cell 11 9-23. (doi:10.1016/j.ccr.2006.10.019)

Thompson SL, Bakhoum SF \& Compton DA 2010 Mechanisms of chromosomal instability. Current Biology 20 R285-R295. (doi:10.1016/j.cub.2010.01.034)

van Gent DC, Hoeijmakers JHJ \& Kanaar R 2001 Chromosomal stability and the DNA double-stranded break connection. Nature Reviews Genetics 2 196-206. (doi:10.1038/35056049)

Venkitaraman AR 2004 Tracing the network connecting brca and fanconi anaemia proteins. Nature Reviews Cancer 4 266-276. (doi:10.1038/nrc1321)

Vries RGJ, Bezrookove V, Zuijderduijn LMP, Kia SK, Houweling A, Oruetxebarria I, Raap AK \& Verrijzer CP 2005 Cancer-associated mutations in chromatin remodeler hSNF5 promote chromosomal instability by compromising the mitotic checkpoint. Genes and Development 19 665-670. (doi:10.1101/gad.335805)

Weaver BAA, Silk AD, Montagna C, Verdier-Pinard P \& Cleveland DW 2007 Aneuploidy acts both oncogenically and as a tumor suppressor. Cancer Cell 11 25-36. (doi:10.1016/j.ccr.2006.12.003)

Wu Z-H 2016 Phenotypes and genotypes of the chromosomal instability syndromes. Translational Pediatrics 5 79-83. (doi:10.21037/ tp.2016.03.04)

Zasadil LM, Britigan EMC, Ryan SD, Kaur C, Guckenberger DJ, Beebe DJ, Moser AR \& Weaver BA 2016 High rates of chromosome missegregation suppress tumor progression but do not inhibit tumor initiation. Molecular Biology of the Cell 27 1981-1989. (doi:10.1091/ mbc.E15-10-0747)

Zhang C-Z, Spektor A, Cornils H, Francis JM, Jackson EK, Liu S, Meyerson M \& Pellman D 2015 Chromothripsis from DNA damage in micronuclei. Nature 522 179-184. (doi:10.1038/nature14493)

Zhou H, Wang T, Zheng T, Teng J \& Chen J 2016 Cep57 is a Mis12interacting kinetochore protein involved in kinetochore targeting of Mad1-Mad2. Nature Communications 7 10151. (doi:10.1038/ ncomms10151)

Received in final form 7 April 2017

Accepted 18 May 2017
(C) 2017 Society for Endocrinology Printed in Great Britain
Published by Bioscientifica Ltd 